\title{
Quantum wrapped giant magnon
}

\author{
Nikolay Gromov, ${ }^{1, *}$ Sakura Schäfer-Nameki, ${ }^{2,+}$ and Pedro Vieira ${ }^{3,+}$ \\ ${ }^{1}$ Service de Physique Théorique, CNRS-URA 2306 C.E.A.-Saclay, F-91191 Gif-sur-Yvette, France; \\ Laboratoire de Physique Théorique de l'Ecole Normale Supérieure et l'Université Paris-VI, Paris, 75231, France; \\ St. Petersburg INP, Gatchina, 188 300, St. Petersburg, Russia \\ ${ }^{2}$ California Institute of Technology, 1200 E California Blvd., Pasadena, California 91125, USA \\ ${ }^{3}$ Laboratoire de Physique Théorique de l'Ecole Normale Supérieure et l'Université Paris-VI, Paris, 75231, France; \\ Departamento de Física e Centro de Física do Porto Faculdade de Ciências da Universidade do Porto Rua do Campo Alegre, \\ 687, 4169-007 Porto, Portugal
}

(Received 24 March 2008; published 31 July 2008)

Understanding the finite-size corrections to the fundamental excitations of a theory is the first step towards completely solving for the spectrum in finite volume. We compute the leading exponential correction to the quantum energy of the fundamental excitation of the light-cone gauged string in $\mathrm{AdS}_{5} \times$ $S^{5}$, which is the giant magnon solution. We present two independent ways to obtain this correction: the first approach makes use of the algebraic curve description of the giant magnon. The second relies on the purely field-theoretical Lüscher formulas, which depend on the world-sheet $S$-matrix. We demonstrate the agreement to all orders in $(\Delta / \sqrt{\lambda})^{-1}$ of these approaches, which, in particular, presents a further test of the $S$-matrix. We comment on generalizations of this method of computation to other string configurations.

DOI: 10.1103/PhysRevD.78.026006

PACS numbers: 11.25.Tq, 11.25.Mj

\section{INTRODUCTION AND SUMMARY}

In the AdS/CFT correspondence we find ourselves at the point where the $S$-matrix of [1-5] is believed to accurately describe the infinite-volume theory, albeit it fails to capture some finite-size effects [6-10]. The first step towards understanding a theory in finite volume is to compute the leading correction to the dispersion relation of its fundamental excitations. These corrections arise through virtual particles circling in the compact direction, which contribute to the self-energy of physical particles [11]. In this paper we compute the leading quantum finite-size corrections to the fundamental excitation of the $\mathrm{AdS}_{5} \times S^{5}$ string theory.

Tree-level light-cone gauged string theory on $\mathrm{AdS}_{5} \times S^{5}$ is a two-dimensional integrable field theory defined on a world-sheet cylinder of length $L / \sqrt{\lambda}$, where $L$ is a large symmetry charge and $1 / \sqrt{\lambda}$, with $\lambda$ the t' Hooft coupling, plays the role of $\hbar$. In the infinite length limit the fundamental excitations are world-sheet solitons, so-called giant magnons (GM) [12], which transform under the residual extended $S U(2 \mid 2)^{2}$ symmetry [3] and have a nonrelativistic dispersion relation ${ }^{1}$

$$
\epsilon_{\infty}(p)=\sqrt{1+\frac{\lambda}{\pi^{2}} \sin ^{2}\left(\frac{p}{2}\right)}
$$

where $p$ is the magnon world-sheet momentum. This

\footnotetext{
*nikgromov@gmail.com

+ss299@theory.caltech.edu

${ }^{\ddagger}$ pedrogvieira@gmail.com

${ }^{1}$ It can also be obtained from a relativistic theory by integrating out some physical degrees of freedom [13].
}

infinite-volume dispersion relation is believed to be exact and almost fixed by symmetry alone [3]. For large $\lambda$,

$$
\epsilon_{\infty}(p)=\frac{\sqrt{\lambda}}{\pi} \sin \left(\frac{p}{2}\right)+0+\mathcal{O}\left(\frac{1}{\sqrt{\lambda}}\right),
$$

where the leading term is the classical energy of the giant magnon and the absence of the $\mathcal{O}(1)$ term means that the first quantum correction (or one-loop shift) vanishes for the magnon in infinite volume. This was explicitly checked in $[14,15]$.

The finite-size corrected dispersion relation differs from the infinite-volume one by exponentially suppressed terms which can be organized according to world-sheet loop order,

$$
\epsilon(p)-\epsilon_{\infty}(p)=\sqrt{\lambda} \delta \epsilon_{\mathrm{cl}}+\delta \epsilon_{1-\text { loop }}+\frac{1}{\sqrt{\lambda}} \delta \epsilon_{2-\text { loop }}+\ldots
$$

The classical finite-size corrections to the dispersion relation were computed in ${ }^{2}[9]$ to be

$$
\begin{aligned}
\delta \epsilon_{\mathrm{cl}}= & -\frac{4}{\pi} \sin ^{3} \frac{p}{2} e^{-(2 \pi \Delta / \sqrt{\lambda} \sin (p / 2))} \\
& +\mathcal{O}\left(e^{-2(2 \pi \Delta / \sqrt{\lambda} \sin (p / 2))}\right),
\end{aligned}
$$

where $\Delta=L+\frac{\sqrt{\lambda}}{\pi} \sin \frac{p}{2}$. The first quantum corrections to the dispersion relation (which will in this case be the leading term since the infinite-volume contribution vanishes) are computed in this paper and are given by (2.13), of

\footnotetext{
${ }^{2}$ This was also preformed in a more controlled orbifold setup in [16] and generalized for bound states of magnons, called dyonic magnons [17], in $[18,19]$.
} 
which the first terms in the $(\Delta / \sqrt{\lambda})^{-1}$ expansion are ${ }^{3}$

$$
\begin{aligned}
\delta \epsilon_{1-\text { loop }}= & \frac{8 \sin ^{2} \frac{p}{4} e^{-(2 \pi \Delta / \sqrt{\lambda})}}{\pi\left(\sin \frac{p}{2}-1\right)\left(\frac{\Delta}{\sqrt{\lambda}}\right)^{1 / 2}} \\
& \times\left[1-\frac{7+4 \sin p-4 \cos p+\sin \frac{p}{2}}{16 \pi\left(\sin \frac{p}{2}-1\right) \frac{\Delta}{\sqrt{\lambda}}}\right. \\
& \left.+\mathcal{O}\left(\frac{1}{\left(\frac{\Delta}{\sqrt{\lambda}}\right)^{2}}\right)\right]+\ldots,
\end{aligned}
$$

where the subleading corrections are of order

$$
\mathcal{O}\left(e^{-(2 \pi \Delta / \sqrt{\lambda} \sin (p / 2))}, e^{-(4 \pi \Delta / \sqrt{\lambda})}\right) .
$$

As will be explained in Sec. II this computation relies solely on the algebraic curve technology [20] and can be easily generalized to other states.

On the other hand it is also known that the leading corrections to the dispersion relation of particles in finite volume are given by Lüscher terms and are a sum of an $F$-term and a $\mu$-term contribution depicted in Fig. 1, which are expressed in terms of the two-particle $S$-matrix. The match with the corrections (1.3) and (1.4) provides therefore an important nontrivial check of the AdS/CFT $S$-matrix [1-5]. The leading classical correction (1.3) was shown to match the $\mu$-term computation $[10,18,19]$ and we shall show that the leading quantum exponential correction (1.4) is accounted for by the $F$-term contribution $[10,11]^{4}$

$$
\begin{aligned}
\delta E_{a}^{F}= & -\int_{\mathbb{R}} \frac{d q}{2 \pi}\left(1-\frac{\epsilon_{\infty}^{\prime}(p)}{\epsilon_{\infty}^{\prime}\left(q^{*}(q)\right)}\right) e^{-i q^{*}(q) L} \\
& \times \sum_{b}(-1)^{F_{b}}\left(S_{b a}^{b a}\left(q^{*}(q), p\right)-1\right),
\end{aligned}
$$

where $p$ and $q$ are the momenta of the magnon that correspond to the physical particle and to the virtual particle propagating in the loop, respectively, and $q^{*}$ is determined by the on-shell condition $q^{2}+\epsilon_{\infty}^{2}\left(q_{*}(q)\right)=0$.

In Sec. III we check that the $F$-term contribution yields the quantum result to all orders in $(L / \sqrt{\lambda})^{-1}$, which agrees with the algebraic curve result that we obtain in Sec. II!

\section{ONE-LOOP ENERGY SHIFT FROM THE ALGEBRAIC CURVE}

\section{A. GM from the algebraic curve}

The algebraic curve formalism [20] maps classical string solutions in $\mathrm{AdS}_{5} \times S^{5}$ to a set of eight quasimomenta

\footnotetext{
${ }^{3}$ Notice that (2.13) is perfectly well behaved for $p=\pi$. The singularity in (1.4) at $p=\pi$ is due to the fact that the saddle point approximation breaks down, because the log branch-points approach the saddle point. The approximation (1.4) of (2.13) is valid as long as $|p-\pi| \gg e^{-\pi \Delta / \sqrt{\lambda}}$.

${ }^{4}$ Note that this formula differs from the one in [10] by the supertrace, i.e. insertion of $(-1)^{F}$ coming from the fermion loops. We will discuss this point in detail in Appendix B.
}
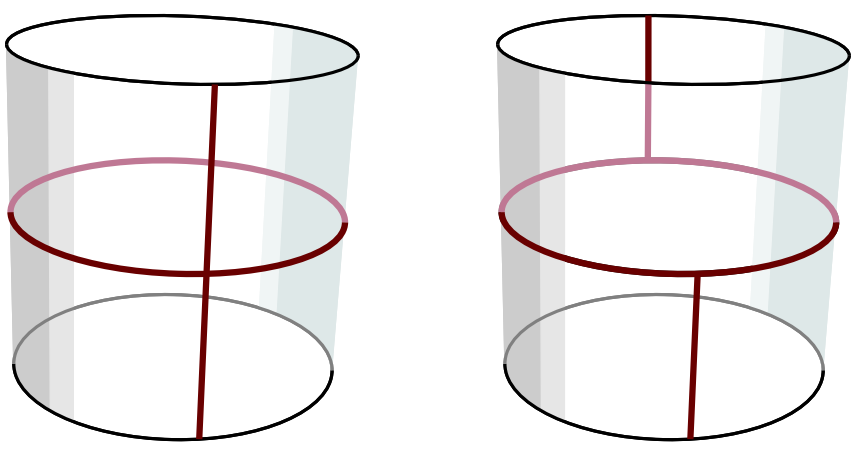

FIG. 1 (color online). The two leading processes contributing to the corrections of the dispersion relation in finite volume: The $F$-term (left-hand side) describes the contribution of a virtual particle loop. We shall see that it accounts for the leading contribution or $\mathcal{O}\left(e^{-2 \pi \Delta / \sqrt{\lambda}}\right)$ correction to the giant magnon one-loop energy. The $\mu$-term (right-hand side) is the effect of a particle splitting into two on-shell particles and computes the classical finite-size effects. It also contributes to the one-loop shift, but since it is $\mathcal{O}\left(e^{-2 \pi \Delta / \sqrt{\lambda} \sin (p / 2)}\right)$, this contribution is subleading.

$\left\{p_{\tilde{1}}, \ldots, p_{\tilde{4}}, p_{\hat{1}}, \ldots, p_{\hat{4}}\right\}$ which define an eight-sheeted Riemann surface. It is convenient to embed the simple giant magnon solution into a family of solutions, called dyonic giant magnons [17], whose infinite-volume dispersion relation is given by

$$
\epsilon_{\infty}^{Q}(p)=\sqrt{Q^{2}+16 g^{2} \sin ^{2}\left(\frac{p}{2}\right)},
$$

where the momentum $p$ of the magnon should obviously not be confused with the quasimomenta $p_{i}$. For $Q=1$ the dyonic magnon reduces to the simple magnon mentioned in the Introduction.

In terms of the variables $X^{ \pm}$which parametrize the magnon momentum as

$$
p=\frac{1}{i} \log \frac{X^{+}}{X^{-}},
$$

and are constrained by

$$
Q=\frac{\sqrt{\lambda}}{4 \pi i}\left(X^{+}+\frac{1}{X^{+}}-X^{-}-\frac{1}{X^{-}}\right),
$$

the infinite-volume dispersion relation takes the form

$$
\epsilon_{\infty}^{Q}=\frac{\sqrt{\lambda}}{4 \pi i}\left(X^{+}-\frac{1}{X^{+}}-X^{-}+\frac{1}{X^{-}}\right) .
$$

The $X^{ \pm}$variables are particularly suitable for the curve treatment described below. Notice that for a single magnon, or more generally for $Q \ll \sqrt{\lambda}$ which is the case we consider in this paper,

$$
X^{ \pm}=e^{ \pm i p / 2}+\mathcal{O}(1 / \sqrt{\lambda}),
$$

and thus $X^{ \pm}=1 / X^{\mp}$. 


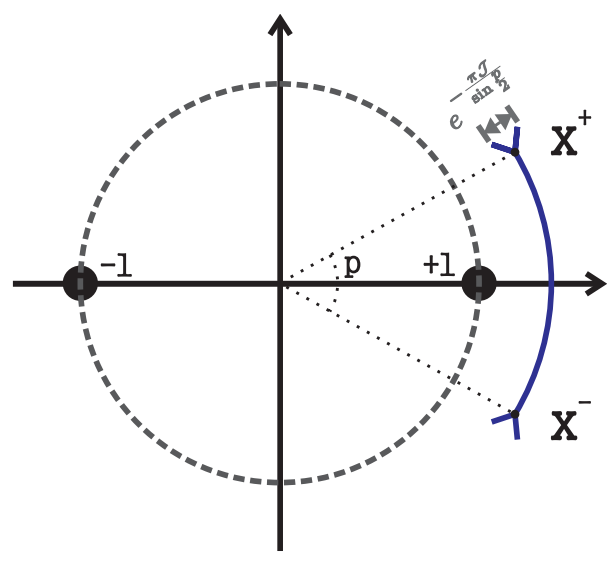

FIG. 2 (color online). The small charge dyonic giant magnon in finite volume [21]: the endpoints of the log-cut between $X^{+}$ and $X^{-}$develop small square root tails, separated by $\mathcal{O}\left(e^{-(\pi \mathcal{J} /(\sin p / 2))}\right)$, which will induce finite-size corrections of this order. For the classical finite-size corrections, these were the only contributions. However, as we explain in the main text, for the leading quantum correction there are more important corrections, which are of order $\mathcal{O}\left(e^{-2 \pi \mathcal{J}}\right)$.

The precise description [21] of the dyonic giant magnon in finite volume is that of a two-cut solution, where the two cuts are very close and thus can be reconnected to form a log-cut condensate with $\log$ branch-points at $X^{ \pm}$, see Fig. 2. The separation of the two branch-points of the two-cut solution is of the $\operatorname{order}^{5} \mathcal{O}\left(e^{-\pi \Delta / \sqrt{\lambda} \sin (p / 2)}\right)$. The classical energy of the giant magnon in finite volume differs from the infinite-volume log result (2.3) by a contribution of the order $[9,19]$,

$$
\mathcal{O}\left(e^{-(2 \pi \Delta / \sqrt{\lambda} \sin (p / 2))}\right) \text {. }
$$

In the same way, if we take into account the precise two-cut structure of the finite volume GM we will also find a contribution to the fluctuation energies-and therefore to the one-loop shift-with the same exponential suppression. However, as already anticipated in the Introduction, there are more important corrections (1.4) of the order

$$
\mathcal{O}\left(e^{-(2 \pi \Delta / \sqrt{\lambda})}\right)
$$

which simply come from the fact that in order to compute the ground state energy in finite volume, we should sum over the fluctuation energy mode number instead of integrating over the momenta of the quantum fluctuations. Thus, to compute the leading-order quantum finite-size corrections we can work with the log-cut description [22] of the giant magnon solution for which [15,22]

\footnotetext{
${ }^{5}$ This result holds for $Q \ll \sqrt{\lambda}$. The generalization for bigger $Q$ is also now [19].
}

$$
\begin{aligned}
p_{\hat{1}, \hat{2}}(x) & =-p_{\hat{\mathfrak{3}, \hat{4}}}(x)=\frac{2 \pi \Delta}{\sqrt{\lambda}} \frac{x}{x^{2}-1} \\
p_{\tilde{2}}(x) & =-p_{\tilde{3}}(x)=\frac{2 \pi \Delta}{\sqrt{\lambda}} \frac{x}{x^{2}-1}+\frac{1}{i} \log \frac{x-X^{+}}{x-X^{-}}+\tilde{\phi}_{2} \\
p_{\tilde{1}}(x) & =-p_{\tilde{4}}(x)=\frac{2 \pi \Delta}{\sqrt{\lambda}} \frac{x}{x^{2}-1}+\frac{1}{i} \log \frac{x-1 / X^{-}}{x-1 / X^{+}}+\tilde{\phi}_{1} .
\end{aligned}
$$

The twists $\tilde{\phi}_{1,2}$ in the quasimomenta [23] are necessary because for a single magnon we cannot satisfy the usual periodic boundary conditions. The usual orbifold treatment $[16,24,25]$ selects the twists

$$
\tilde{\phi}_{1}=\tilde{\phi}_{2}=-p / 2
$$

as reviewed in Appendix C.

We shall now study the quantum fluctuations around this classical solution. By perturbing the quasimomenta via the addition of extra poles we can compute the fluctuation energies $\Omega_{n}^{i j}$ around this classical solution [26]. The poles are shared by quasimomenta $p_{i}$ and $p_{j}$ and the different possible choices of quasimomenta correspond to different string polarizations, namely

$$
\begin{array}{r}
S^{5}:(\tilde{1} \tilde{3}),(\tilde{1} \tilde{4}),(\tilde{2} \tilde{3}),(\tilde{2} \tilde{4}), \\
\operatorname{AdS}^{5}:(\hat{1} \hat{3}),(\hat{1} \hat{4}),(\hat{2} \hat{3}),((\hat{2} \hat{4}), \\
\text { Fermions: }(\hat{1} \tilde{3}),(\hat{1} \tilde{4}),(\hat{2} \tilde{3}),((\hat{2} \tilde{4}), \\
(\tilde{1} \hat{3}),(\tilde{1} \hat{4}),(\tilde{2} \hat{3}),(\tilde{2} \hat{4}) .
\end{array}
$$

Moreover each quantum fluctuation has a mode number $n$, which will dictate where the corresponding pole outside the unit circle will be located through

$$
p_{i}\left(x_{n}\right)-p_{j}\left(x_{n}\right)=2 \pi n .
$$

In this way we can find the semiclassical spectrum of fluctuations around the GM solution which reads

$$
\delta \Delta=\sum_{(i j), n} N_{n}^{i j} \Omega_{n}^{i j} .
$$

The result of the perturbation of the quasimomenta, which is explicitly presented in Appendix B, is that all fluctuation energies are given by the same function, when expressed in terms of the position of the poles $x_{n}$, that is $\Omega_{n}^{i j}=\Omega\left(x_{x}^{i j}\right)$ for all $i j$. The function $\Omega(x)$ is given by

$$
\Omega(x)=\frac{2}{x^{2}-1}\left(1-\frac{X^{+}+X^{-}}{X^{+} X^{-}+1} x\right) .
$$

Notice that this does not mean that the fluctuation energies are all the same, when expressed in terms of the mode number $n$, because for each string polarization $i j$ the map $x \leftrightarrow n$ is different and given by (2.8).

Formula (2.10) for the fluctuation energies has a nice physical interpretation which can indeed be used as an 
alternative (and simpler) derivation of this expression. Namely, we know $[20,26]$ that the quantum fluctuations located at $x$ in the algebraic curve carry energy and momentum ${ }^{6}$

$$
\epsilon_{\text {fluct }}(x)=\frac{2}{x_{n}^{2}-1}, \quad p_{\text {fluct }}(x)=\frac{x}{x_{n}^{2}-1} .
$$

When we add a fluctuation the energy is not simply given by $\epsilon_{\text {fluct }}(x)$ because the fluctuation will backreact and slightly modify the classical background. The correct energy shift $\Omega$ is then given by the energy of the fluctuation plus the backreaction of the the giant magnon,

$$
\Omega(x)=\epsilon_{\text {fluct }}(x)+\left(\epsilon_{\infty}^{Q}(p)\right)^{\prime} \delta p,
$$

where $\delta p$ is the shift in the momentum of the magnon due to the presence of the fluctuation, and by momentum conservation it equals $-\delta p_{\text {fluct }}(x)$. Since

$$
\left(\epsilon_{\infty}^{Q}(p)\right)^{\prime}=2 \frac{X^{+}+X^{-}}{X^{+} X^{-}+1},
$$

formula (2.10) follows.

\section{B. GM one-loop shift}

The ground state energy around a classical solution, known as the one-loop energy shift, is given by the graded sum over one half of each fluctuation energy:

$$
\delta \epsilon_{1-\mathrm{loop}}=\frac{1}{2} \sum_{n \in \mathbb{Z}} \sum_{(i j)}(-1)^{F_{i j}} \Omega_{n}^{i j} .
$$

The sum $(i j)$ extends over all polarizations as listed in (2.7). It is convenient $[7,27]$ to rewrite this sum over $n$ as an integral

$$
\delta \in_{1-\text { loop }}=-\sum_{(i j)}(-1)^{F_{i j}} \oint_{\mathbb{R}} \frac{d n}{4 i} \chi \mathrm{O} \tau(\pi n) \Omega\left(x_{n}^{i j}\right),
$$

where the contour encircles the real axis. Next, for each polarization $(i j)$ we change the variables to $x$ via (2.8) so that the integration over $n$ maps to contours which encircle the fluctuation positions $x_{n}^{i j}$ located outside the unit circle $\mathbb{U}$. For a generic polarization $(i j)$, we can deform this contour $^{7}$ in the $x$ plane and obtain an integral over the unit circle plus an integral over the eventual cuts of the classical solution, in this case the log-cut in (2.5). Up to exponentially suppressed terms, which we are now focussing on, these two contributions were universally analyzed

\footnotetext{
${ }^{6}$ An extra excitation is still described by formulas (2.1), (2.2), and (2.3) with $Q=1$. Quantum fluctuations in the scaling limit have momentum $q \sim \mathcal{O}(1 / \sqrt{\lambda})$ so that $X_{q}^{ \pm}$are then given by $X_{q}^{ \pm}=x \pm \frac{4 \pi}{\sqrt{\lambda}} \frac{x^{2}}{x^{2}-1}$, where $x$ is the position of the quantum fluctuation in the algebraic curve (or the position of the Bethe roots in the AdS/CFT Bethe ansatz [2]). Thus in this limit (2.11) follows from (2.1) and (2.3).

${ }^{7}$ Notice that the orientation of the contour flips in the process.
}

in $[23,27]$ respectively. The possible contribution from the integral over the log-cut is given by the log branch-points $x=X^{ \pm}$, which become pole terms when plugged into the integral in the $x$ plane (see (2.13) below). It is easy to see that this contribution enters as

$$
\mathcal{O}\left(e^{-(2 \pi \Delta / \sqrt{\lambda} \sin (p / 2))}\right)
$$

and is subleading compared with the contribution coming from the integral over the unit circle which we will now consider. We shall however return to this point in the discussions.

We therefore have

$$
\begin{aligned}
\delta \in_{1-\text { loop }}= & -\sum_{(i j)}(-1)^{F_{i j}} \oint_{\hat{U}} \frac{d x}{4 i} \frac{p_{i}^{\prime}-p_{j}^{\prime}}{2 \pi} \\
& \times \cot \left(\frac{p_{i}-p_{j}}{2}\right) \Omega(x) .
\end{aligned}
$$

On the upper/lower half of the unit circle, $\mathbb{U}^{ \pm}$, the quasimomenta are large and

$$
\cot \left(\frac{p_{i}-p_{j}}{2}\right)= \pm i\left(1+2 e^{\mp i\left(p_{i}-p_{j}\right)}+\ldots\right) .
$$

Picking the leading term we get a zero result for the oneloop shift since

$$
\sum_{i j}(-1)^{F_{i j}}\left(p_{i}^{\prime}-p_{j}^{\prime}\right)=0,
$$

which is precisely the vanishing result derived in [22] and mentioned in the Introduction in (1.2). The leading exponential correction to the one-loop shift-which in this case turns out to be the leading result for this quantity-is obtained by picking the second term of the expansion of the cotangent. After an integration by parts we find ${ }^{8}$

$$
\delta \in_{1-\text { loop }}=\oint_{\mathbb{U}^{+}} \frac{d x}{2 \pi i} \partial_{x} \Omega(x) \sum_{(i j)}(-1)^{F_{i j}} e^{-i\left(p_{i}-p_{j}\right)},
$$

where $\Omega(x)$ is given by (2.10). From (2.5) using that for a single giant magnon $1 / X^{\mp}=X^{ \pm}$including the twists given in (2.6), we obtain

$$
\begin{aligned}
\sum_{(i j)}(-1)^{F_{i j}} e^{-i\left(p_{i}-p_{j}\right)}= & \left(2 \frac{x-X^{-}}{x-X^{+}} e^{i p / 2}-2\right)^{2} \\
& \times \exp \left(-\frac{i 4 \pi \Delta}{\sqrt{\lambda}} \frac{x}{x^{2}-1}\right) .
\end{aligned}
$$

It is clear that this expression is suppressed by $e^{-2 \pi \Delta / \sqrt{\lambda}}$ which is indeed leading compared to the effects we have discarded so far. The expansion (1.4) mentioned in the

\footnotetext{
${ }^{8}$ The extra factor of 2 appearing comes from keeping the integration over the upper half circle only.
} 
introduction is trivially obtained by expanding around the saddle point $x \simeq i$.

\section{LÜSCHER $F$-TERM}

The Lüscher $F$-term is given by (1.5). We will now evaluate this for a single magnon and show that it gives rise to the same one-loop leading exponential correction to all orders in $(\Delta / \sqrt{\lambda})^{-1}$ as in (2.13). Physically, the $F$-term computes the correction to the energy of the giant magnon with momentum $p$ by a virtual particle with momentum $q$ circulating in the compact world-sheet direction. Because of the supertrace in (1.5), whose origin we discuss in Appendix B, the only contribution comes from the $S$-matrix part.

The momenta of both the physical particle, $p$, and the virtual particle, $q^{*}$, appear in the $S$-matrix through $X_{p}^{ \pm} \equiv$ $X^{ \pm}$and $X_{q^{*}}^{ \pm}$. As mentioned before, the former lie in the unit circle with

$$
X^{ \pm}=e^{ \pm i p / 2}+\mathcal{O}(1 / \sqrt{\lambda}) .
$$

As for the virtual particle [10] $q^{2}+\epsilon_{\infty}^{2}\left(q_{*}(q)\right)=0$ so that for $q \sim \mathcal{O}(1)$ we have $q^{*} \sim \mathcal{O}(1 / \sqrt{\lambda})$ and thus

$$
X_{q^{*}}^{+}=x+\mathcal{O}(1 / \sqrt{\lambda}) .
$$

In terms of $x$ we have

$$
q_{*}=\frac{4 \pi}{\sqrt{\lambda}} \frac{x}{x^{2}-1}, \quad q=i \frac{x^{2}+1}{x^{2}-1} .
$$

The contour over the real $q$ axis is mapped to the upper half of the unit circle in the $x$ plane and the saddle point of the $F$-term, at $q=0$, is mapped to $x=i$, which is the same saddle point we found at the end of the previous section.

Moreover,

$$
1-\frac{\epsilon_{\infty}^{\prime}(p)}{\epsilon_{\infty}^{\prime}\left(q^{*}(q)\right)}=1-\frac{x^{2}+1}{2 x} \frac{X^{+}+X^{-}}{X^{+} X^{-}+1},
$$

so that the $F$-term (1.5) becomes

$$
\begin{aligned}
\delta \in_{a}^{F}= & \oint_{\mathbb{U}^{+}} \frac{d x}{2 \pi i} \partial_{x} \Omega(x) e^{-4 \pi(i J / \sqrt{\lambda})\left(x / x^{2}-1\right)} \\
& \times \sum_{b}(-1)^{F_{b}} S_{b a}^{b a}\left(q^{*}(q), p\right),
\end{aligned}
$$

where $\Omega(x)$ is the same function (2.10) which appeared in the curve computation! Finally, using the explicit form of the AdS/CFT $S$-matrix in this setup, which we discuss in Appendix B, we find that this contributes for the particular kinematics (3.1) and (3.2) as

$$
\begin{aligned}
\sum_{b}(-1)^{F_{b}} S_{b a}^{b a}\left(q^{*}(q), p\right)= & e^{-4 \pi(i(\Delta-J) / \sqrt{\lambda})\left(x /\left(x^{2}-1\right)\right)} \\
& \times\left(2 \frac{x-X^{-}}{x-X^{+}} \sqrt{\frac{X^{+}}{X^{-}}}-2\right)^{2} .
\end{aligned}
$$

Thus, combining (3.3) and (3.4) we find precisely the integral (2.13) appearing in the curve computation. Since our match is at the level of the integrals we have an all order (in $\left.(\Delta / \sqrt{\lambda})^{-1}\right)$ agreement between both computations!

\section{DISCUSSION AND FUTURE WORK}

The ground state energy for the giant magnon in finite volume is an exponentially suppressed quantity which appears to have an expansion

$$
\begin{aligned}
\delta \epsilon_{1 \text {-loop }}= & a_{1,0} e^{-(2 \pi \Delta / \sqrt{\lambda})} \\
& +\sum_{n \geq 0, m \geq 1}^{\infty} a_{n, m} e^{-n(2 \pi \Delta / \sqrt{\lambda})-m(2 \pi \Delta / \sqrt{\lambda} \sin (p / 2))},
\end{aligned}
$$

where each prefactor $a_{n, m}$ is a nontrivial function of $\Delta=$ $L+\sqrt{\lambda} \sin \frac{p}{2}$ and $p$. We computed the leading contribution, namely $a_{1,0}(\Delta, p)$, and checked that it can be reproduced through an $F$-term contribution (cf. Fig. 1).

Depending on the value of the momentum $p$, the next-toleading order coefficient is $a_{2,0}$ or $a_{0,1}$. The latter has the typical exponential suppression we find from the $\mu$ term computation [10] and will surely come from the next-toleading correction to the results in [10]. From the algebraic curve point of view this correction could come from two different places: first, from the discrepancy between the log-cut description [22] used here and the true giant magnon in finite volume described in [21], which is in fact a two-cut solution (cf. Fig. 2); second, from the integral of the fluctuation energies over the cuts of the classical solution as discussed in Sec. II B. It would be very interesting to match the curve and the $\mu$-term results.

As for the the coefficients $a_{n, 0}$, they come from the subleading terms in the expansion (2.12) of the cotangent, and therefore can be trivially computed from the curve point of view to be

$$
\begin{aligned}
a_{n, 0} e^{-(2 \pi n \Delta \sqrt{\lambda})}= & \oint_{\mathbb{U}^{+}} \frac{d x}{2 \pi i} \partial_{x} \Omega(x) \sum_{(i j)}(-1)^{F_{i j}} \\
& \times e^{-i n\left(p_{i}-p_{j}\right)}
\end{aligned}
$$

where $\mathbb{U}^{+}$is the upper half of the unit circle, $p_{i}$ the giant magnon quasimomenta (2.5) and $\Omega(x)$ the fluctuation energies (2.10).

The remaining coefficients $a_{n, m}$ will most likely come from virtual processes with $n$ virtual particle loops in addition to $m$ splits into two on-shell particles. It would be extremely instructive to generalize the $\mu$ - and $F$-term results of Lüscher to allow for such processes. The curve computation might serve as an important guide since in principle these exponential corrections can be independently computed in this formalism. It would be interesting to try to find a more general integrable structure behind 
these contributions, eventually using the already known $a_{n, 0}$ as potential guidelines.

Another possible direction would be to compute the two (world-sheet) loop correction to the dispersion relation $\epsilon_{\infty}(p)$ of the giant magnon. The leading-order term will be of the order $\mathcal{O}\left(e^{-2 \pi \Delta / \sqrt{\lambda}}\right)$ and will come from the nextto-leading expansion in $1 / \sqrt{\lambda}$ of the $F$-term obtained in this paper. This computation would provide a two-loop prediction for the dispersion relation of the giant magnon in finite volume which would be interesting to check against a direct two-loop computation in the spirit of [28].

Yet another interesting next step is to consider more physical string solutions with zero total momentum. The simplest example is to consider two magnons with momenta $p$ and $-p$ (this setup was studied classically in [19]). The computations in the sections above can be trivially generalized both in the curve and in the $F$-term setup and yield for the leading contribution

$$
\delta \in_{1-\text { loop }}^{2 \text { magnons }}=\frac{128 \sin ^{2} \frac{p}{2}}{\pi i} \oint_{\mathbb{U}^{+}} \frac{d x x^{3} \exp \left(-\frac{i 4 \pi \Delta}{\sqrt{\lambda}} \frac{x}{x^{2}-1}\right)}{\left(x^{2}-1\right)^{2}\left(x^{2}-2 i x \sin \frac{p}{2}-1\right.},
$$

for the one-loop shift of the two magnon system in finite volume. Here $\Delta-L=2 \frac{\sqrt{\lambda}}{\pi} \sin \frac{p}{2}$. As an expansion in large $\Delta / \sqrt{\lambda}$ we find therefore the leading terms:

$$
\delta \epsilon_{1-\text { loop }}^{2 \text { magnons }}=-\frac{8 \sin ^{2} \frac{p}{2}}{\pi\left(1-\sin \left(\frac{p}{2}\right)\right)^{2}} \frac{e^{-(2 \pi \Delta / \sqrt{\lambda})}}{\left(\frac{\Delta}{\sqrt{\lambda}}\right)^{1 / 2}}\left[1+\frac{\left(11-3 \sin \left(\frac{p}{2}\right)\right)}{16 \pi\left(1-\sin \left(\frac{p}{2}\right)\right)} \frac{1}{\frac{\Delta}{\sqrt{\lambda}}}+O\left(\frac{1}{\left(\frac{\Delta}{\sqrt{\lambda}}\right)^{2}}\right)\right] .
$$

Finally it would be interesting to try to extend our computations to the most general setup possible including, not only the more general dyonic giant magnons, but also generic classical solutions. For solutions moving in $S^{5}$, when $L$ is much larger than all the other filling fractions the leading exponential corrections will behave like

$$
\mathcal{O}\left(e^{-(2 \pi \Delta / \sqrt{\lambda})}\right)
$$

since the sum over frequencies, when transformed into an integral in the $x$ plane, will be dominated by a saddle point at $x \simeq i$ where the quasimomenta are, to leading order,

$$
p_{i} \simeq \pm \frac{2 \pi \Delta}{\sqrt{\lambda}} \frac{x}{x^{2}-1}
$$

This is in agreement with the findings in [7]. We shall address these issues in a forthcoming publication [29] where we study the $F$-term vs curve approach for generic classical solutions.

\section{ACKNOWLEDGMENTS}

We would like to thank T. Dimofte, A. Mikhailov, J. Minahan, R. Janik, V. Kazakov, and K. Zarembo for many useful discussions. The work of N. G. was partially supported by RSGSS-1124.2003.2 and by RFFI Project Grant No. 06-02-16786. N. G. was also partly supported by ANR Grant INT-AdS/CFT (Contract No. ANR36ADSCSTZ). We would like to thank the Isaac Newton Institute in Cambridge for an inspiring atmosphere during the SIS workshop.

\section{APPENDIX A: DETAILS FOR ALGEBRAIC CURVE COMPUTATION}

To find the fluctuation energies we perturb the quasimomenta (2.5) so that $p_{i}(x) \rightarrow p_{i}(x)+\delta p_{i}(x)$. The perturbation is fixed by some simple analytical properties of $\delta p_{i}(x)$. We add $N_{n}^{i j}$ fluctuations with mode number $n$ and polarization $(i j)$. This means $\delta p_{i}(x)$ must have poles at position

$$
p_{i}\left(x_{n}^{i j}\right)-p_{j}\left(x_{n}^{i j}\right)=2 \pi n,
$$

with residue

$$
\delta p_{i}(x) \sim \eta_{i} N_{n}^{i j} \frac{\alpha\left(x_{n}^{i j}\right)}{x-x_{n}^{i j}},
$$

where the signs of the residues are

$$
\begin{aligned}
\eta_{\hat{1}} & =\eta_{\hat{2}}=\eta_{\tilde{3}}=\eta_{\tilde{4}}=-\eta_{\hat{3}}=-\eta_{\hat{4}} \\
& =-\eta_{\tilde{1}}=-\eta_{\tilde{1}}=1,
\end{aligned}
$$

and

$$
\alpha(x) \equiv \frac{4 \pi}{\sqrt{\lambda}} \frac{x^{2}}{x^{2}-1} .
$$

Analogously to the original quasimomenta, the fluctuation $\delta p_{i}(x)$ can have poles at $x= \pm 1$ but those must be synchronized,

$$
\begin{gathered}
\left\{\delta p_{\hat{1}}, \delta p_{\hat{2}}, \delta p_{\hat{3}}, \delta p_{\hat{4}}, \delta p_{\tilde{1}}, \delta p_{\tilde{2}}, \delta p_{\tilde{3}}, \delta p_{\tilde{4}}\right\} \\
\simeq \frac{\left\{\alpha_{ \pm}, \alpha_{ \pm}, \beta_{ \pm}, \beta_{ \pm} \mid \alpha_{ \pm}, \alpha_{ \pm}, \beta_{ \pm}, \beta_{ \pm}\right\}}{x \pm 1},
\end{gathered}
$$

due to the Virasoro constraints. There is also an $x \rightarrow 1 / x$ symmetry inherited from the coset structure of the connection, which for the fluctuations imposes 


$$
\delta p_{\hat{1}, \hat{4}}(x)=-\delta p_{\hat{2}, \hat{3}}(1 / x), \quad \delta p_{\tilde{1}, \tilde{4}}(x)=-\delta p_{\tilde{2}, \tilde{3}}(1 / x) .
$$

The large $x$ asymptotics of the quasimomenta are fixed by the global charges of the theory and are

$$
\left(\begin{array}{l}
\delta p_{\hat{1}} \\
\delta p_{\hat{2}} \\
\delta p_{\hat{3}} \\
\delta p_{\hat{4}} \\
\delta p_{\tilde{1}} \\
\delta p_{\tilde{2}} \\
\delta p_{\tilde{3}} \\
\delta p_{\tilde{4}}
\end{array}\right) \simeq \frac{1}{x} \frac{4 \pi}{\sqrt{\lambda}}\left(\begin{array}{lll}
+\delta \Delta / 2 & +N_{\hat{1} \hat{4}}+N_{\hat{1} \hat{3}}+N_{\hat{1} \tilde{3}}+N_{\hat{1} \tilde{4}} \\
+\delta \Delta / 2 & +N_{\hat{2} \hat{3}}+N_{\hat{2} \hat{4}}+N_{\hat{2} \tilde{4}}+N_{\hat{2} \tilde{3}} \\
-\delta \Delta / 2 & -N_{\hat{2} \hat{3}}-N_{\hat{1} \hat{3}}-N_{\tilde{1} \hat{3}}-N_{\tilde{2} \hat{3}} \\
-\delta \Delta / 2 & -N_{\hat{1} \hat{4}}-N_{\hat{2} \hat{4}}-N_{\tilde{2} \hat{4}}-N_{\tilde{1} \hat{4}} \\
& -N_{\tilde{1} \tilde{4}}-N_{\tilde{1} \tilde{3}}-N_{\tilde{1} \hat{3}}-N_{\tilde{1} \hat{4}} \\
& -N_{\tilde{2} \tilde{3}}-N_{\tilde{2} \tilde{4}}-N_{\tilde{2} \hat{4}}-N_{\tilde{2} \hat{3}} \\
& +N_{\tilde{2} \tilde{3}}+N_{\tilde{1} \tilde{3}}+N_{\hat{1} \tilde{3}}+N_{\hat{2} \tilde{3}} \\
& +N_{\tilde{1} \tilde{4}}+N_{\tilde{2} \tilde{4}}+N_{\hat{2} \tilde{4}}+N_{\hat{1} \tilde{4}}
\end{array}\right) .
$$

Finally the fluctuations will backreact onto the original solution thus shifting the log-cut. Therefore close to $X^{ \pm}$ the quasimomenta will behave like

$$
\begin{aligned}
& \delta p_{\tilde{2}, \tilde{3}}(x) \sim \partial \log \left(x-X^{ \pm}\right) \sim \frac{1}{x-X^{ \pm}}, \\
& \delta p_{\tilde{1}, \tilde{4}}(x) \sim \frac{1}{x-1 / X^{ \pm}} .
\end{aligned}
$$

This is the only particularity of the GM when compared to the solutions studied in [26] where close to the branchpoints $x^{0}$ of the classical solution we had $\delta p_{i}(x) \sim$ $\partial \sqrt{x-x_{0}} \sim \frac{1}{\sqrt{x-x_{0}}}$.

The analytical properties (A1)-(A5) are obviously enough to fix the functions $\delta p_{i}(x)$ completely-the steps involved in mimicking the ones in [26] closely. We find

$$
\begin{aligned}
& \delta p_{\tilde{1}}=+\frac{A x+B}{x^{2}-1}-\sum_{n, j=\tilde{3} \tilde{4} \hat{3} \hat{4}}\left(\frac{N_{n}^{\tilde{1} j} \alpha\left(x_{n}^{\tilde{1} j}\right)}{x-x_{n}^{\tilde{1} j}}-\frac{N_{n}^{\tilde{2} j} \alpha\left(x_{n}^{\tilde{2} j}\right)}{1 / x-x_{n}^{\tilde{j} j}}-\frac{N_{n}^{\tilde{2} j} \alpha\left(x_{n}^{\tilde{2} j}\right)}{x_{n}^{\tilde{j} j}}\right)-\sum_{\beta= \pm}\left(\frac{A^{\beta}}{1 / x-X^{\beta}}+\frac{A^{\beta}}{X^{\beta}}\right) \\
& \delta p_{\tilde{2}}=+\frac{A x+B}{x^{2}-1}-\sum_{n, j=\tilde{3} \tilde{4} \hat{3} \hat{4}}\left(\frac{N_{n}^{\tilde{2} j} \alpha\left(x_{n}^{\tilde{2} j}\right)}{x-x_{n}^{\tilde{2} j}}-\frac{N_{n}^{\tilde{1} j} \alpha\left(x_{n}^{\tilde{1} j}\right)}{1 / x-x_{n}^{\tilde{1} j}}-\frac{N_{n}^{\tilde{1} j} \alpha\left(x_{n}^{\tilde{1} j}\right)}{x_{n}^{\tilde{j} j}}\right)+\sum_{\beta= \pm} \frac{A^{\beta}}{x-X^{\beta}} \\
& \delta p_{\tilde{3}}=-\frac{C x+D}{x^{2}-1}+\sum_{n, j=\tilde{1} \tilde{2} \hat{\imath} \hat{2}}\left(\frac{N_{n}^{\tilde{3} j} \alpha\left(x_{n}^{\tilde{3} j}\right)}{x-x_{n}^{\tilde{3} j}}-\frac{N_{n}^{\tilde{4} j} \alpha\left(x_{n}^{\tilde{4} j}\right)}{1 / x-x_{n}^{\tilde{4} j}}-\frac{N_{n}^{\tilde{4} j} \alpha\left(x_{n}^{\tilde{4} j}\right)}{x_{n}^{\tilde{4} j}}\right)-\sum_{\beta= \pm} \frac{A^{\beta}}{x-X^{\beta}} \\
& \delta p_{\tilde{4}}=-\frac{C x+D}{x^{2}-1}+\sum_{n, j=\tilde{1} \tilde{2} \hat{1} \hat{2}}\left(\frac{N_{n}^{\tilde{4} j} \alpha\left(x_{n}^{\tilde{4} j}\right)}{x-x_{n}^{\tilde{4} j}}-\frac{N_{n}^{\tilde{3} j} \alpha\left(x_{n}^{\tilde{3} j}\right)}{1 / x-x_{n}^{\tilde{3} j}}-\frac{N_{n}^{\tilde{3} j} \alpha\left(x_{n}^{\tilde{3} j}\right)}{x_{n}^{\tilde{3} j}}\right)+\sum_{\beta= \pm}\left(\frac{A^{\beta}}{1 / x-X^{\beta}}+\frac{A^{\beta}}{X^{\beta}}\right) \\
& \delta p_{\hat{1}}=+\frac{A x+B}{x^{2}-1}+\sum_{n, j=\hat{3} \hat{3} \tilde{3} \tilde{4}}\left(\frac{N_{n}^{\hat{1} j} \alpha\left(x_{n}^{\hat{1} j}\right)}{x-x_{n}^{\hat{1} j}}-\frac{N_{n}^{\hat{2} j} \alpha\left(x_{n}^{\hat{2} j}\right)}{1 / x-x_{n}^{\hat{2} j}}-\frac{N_{n}^{\hat{2} j} \alpha\left(x_{n}^{\hat{2} j}\right)}{x_{n}^{\hat{2} j}}\right) \\
& \delta p_{\hat{2}}=+\frac{A x+B}{x^{2}-1}+\sum_{n, j=\hat{3} \hat{4} \tilde{3} \tilde{4}}\left(\frac{N_{n}^{\hat{2} j} \alpha\left(x_{n}^{\hat{2} j}\right)}{x-x_{n}^{\hat{2} j}}-\frac{N_{n}^{\hat{1} j} \alpha\left(x_{n}^{\hat{1} j}\right)}{1 / x-x_{n}^{\hat{1} j}}-\frac{N_{n}^{\hat{1} j} \alpha\left(x_{n}^{\hat{1} j}\right)}{x_{n}^{\hat{1} j}}\right) \\
& \delta p_{\hat{3}}=-\frac{C x+D}{x^{2}-1}-\sum_{n, j=\hat{1} \hat{2} \tilde{1} \tilde{2}}\left(\frac{N_{n}^{\hat{3} j} \alpha\left(x_{n}^{\hat{3} j}\right)}{x-x_{n}^{\hat{j} j}}-\frac{N_{n}^{\hat{4} j} \alpha\left(x_{n}^{\hat{4} j}\right)}{1 / x-x_{n}^{\hat{4} j}}-\frac{N_{n}^{\hat{4} j} \alpha\left(x_{n}^{\hat{4} j}\right)}{x_{n}^{\hat{4} j}}\right) \\
& \delta p_{\hat{4}}=-\frac{C x+D}{x^{2}-1}-\sum_{n, j=\hat{1} \hat{2} \tilde{1} \tilde{2}}\left(\frac{N_{n}^{\hat{4} j} \alpha\left(x_{n}^{\hat{4} j}\right)}{x-x_{n}^{\hat{4} j}}-\frac{N_{n}^{\hat{3} j} \alpha\left(x_{n}^{\hat{3} j}\right)}{1 / x-x_{n}^{\hat{3} j}}-\frac{N_{n}^{\hat{3} j} \alpha\left(x_{n}^{\hat{j} j}\right)}{x_{n}^{\hat{3} j}}\right)
\end{aligned}
$$

where the $x \rightarrow 1 / x$ symmetry fixes

$$
\begin{aligned}
& B=-\sum_{\beta= \pm} \frac{A^{\beta}}{X^{\beta}}+\sum_{i=\tilde{1} \tilde{2}, j=\hat{3} \hat{4} \tilde{3} \tilde{4}} N_{n}^{i j} \frac{\alpha\left(x_{n}^{i j}\right)}{x_{n}^{i j}}=-\sum_{i=\hat{1} \hat{2}, j=\tilde{3} \tilde{4} \hat{3} \hat{4}} N_{n}^{i j} \frac{\alpha\left(x_{n}^{i j}\right)}{x_{n}^{i j}} \\
& D=-\sum_{\beta= \pm} \frac{A^{\beta}}{X^{\beta}}+\sum_{i=\tilde{3} \tilde{4}, j=\hat{1} \hat{2} \tilde{1} \tilde{2}} N_{n}^{i j} \frac{\alpha\left(x_{n}^{i j}\right)}{x_{n}^{i j}}=-\sum_{i=\hat{3} \hat{4}, j=\tilde{1} \tilde{2} \hat{1} \hat{2}} N_{n}^{i j} \frac{\alpha\left(x_{n}^{i j}\right)}{x_{n}^{i j}}
\end{aligned}
$$

while the large $x$ asymptotics yields 


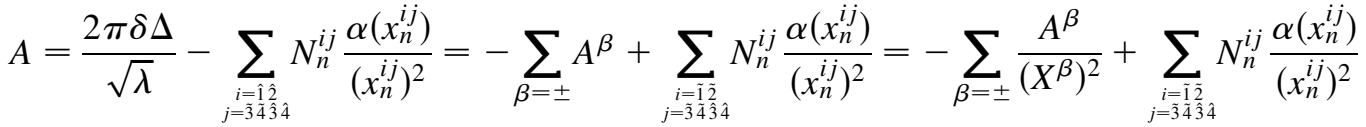

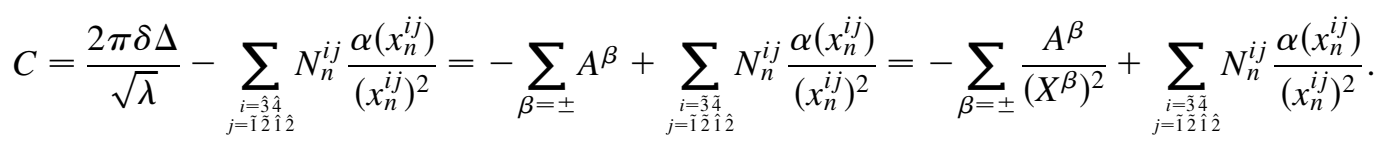

These conditions completely fix $A, B, C, D, A^{ \pm}, \delta \Delta$ and require moreover $\sum_{n, i j} n N_{n}^{i j}=0$ which is nothing but the string level matching condition. For $\delta \Delta$ the result is given by (2.9) with $\Omega(x)$ as in (2.10).

\section{APPENDIX B: DETAILS FOR $F$-TERM COMPUTATION}

We now specify all the terms appearing in the $F$-term integral (1.5). For our purposes it is enough to consider the Arutyunov-Frolov-Staudacher part [30] of the $S$-matrix [35]. The one-loop Hernandez-Lopez correction would contribute one order higher in $1 / \sqrt{\lambda}$.

We should now also comment on the $(-1)^{F}$ insertion in (1.5). In theories with bosonic and fermionic degrees of freedom, the self-energy corrections, which are computed by the Lüscher formulas, enter with different signs depending on the statistics of the particles in the loop. This arises solely through the fermion contractions and was also observed in applications of the Lüscher formula to chiral perturbation theory in [31] - see e.g. formula (10) in the first reference in [31]. In the self-energy diagrams $I_{a b c}, J_{a b c}$ and $K_{a b}$ of [10,11], we have external lines $a$ given by the giant magnon, so that the statistics of the internal contributions can be summarized by $(-1)^{F_{b}}$. This results, in particular, in the insertion of $(-1)^{F_{b}}$ in (1.5) and the contribution from the $S$-matrix is then ${ }^{9}$ :

$$
\begin{aligned}
\sum_{b}(-1)^{F_{b}} S_{b a}^{b a}\left(q^{*}, p\right)= & \left(2 a_{1}+a_{2}-2 a_{6}\right)^{2} \frac{x_{q^{*}}^{-}-x_{p}^{+}}{x_{q^{*}}^{+}-x_{p}^{-}} \\
& \times \frac{1-\frac{1}{x_{q^{*}}^{+}}}{1-\frac{1}{x_{q^{*}}^{-} x_{p}^{+}}} \sigma^{2}\left(x_{q^{*}}, x_{p}\right),
\end{aligned}
$$

where

$$
\begin{gathered}
a_{1}=\frac{x_{p}^{-}-x_{q^{*}}^{+}}{x_{p}^{+}-x_{q^{*}}^{-}} \sqrt{\frac{x_{p}^{+}}{x_{q}^{-}}} \sqrt{\frac{x_{q^{*}}^{-}}{x_{q^{*}}^{+}}}, \quad a_{6}=\frac{x_{q^{*}}^{+}-x_{p}^{+}}{x_{q^{*}}^{-}-x_{p}^{+}} \sqrt{\frac{x_{q^{*}}^{-}}{x_{q^{*}}^{+}}} \quad a_{2}=\frac{\left(x_{q^{*}}^{-}-x_{q^{*}}^{+}\right)\left(x_{p}^{-}-x_{p}^{+}\right)\left(x_{p}^{-}+x_{q^{*}}^{+}\right)}{\left(x_{q^{*}}^{-}-x_{p}^{+}\right)\left(x_{q^{*}}^{-} x_{p}^{-}-x_{q^{*}}^{+} x_{p}^{+}\right)} \sqrt{\frac{x_{p}^{+}}{x_{q}^{-}}} \sqrt{\frac{x_{q^{*}}^{-}}{x_{q^{*}}^{+}}} \\
\sigma^{2}\left(x_{q^{*}}, x_{p}\right)=\left(\frac{1-\frac{1}{x_{p}^{-} x_{q^{*}}^{+}}}{1-\frac{1}{x_{q^{*}}^{-} x_{p}^{+}}}\right)^{-2}\left(\frac{1-\frac{1}{x_{p}^{-} x_{q^{*}}^{+}}}{1-\frac{1}{x_{q^{*}}^{-}}} \frac{1-\frac{1}{x_{p}^{-}}}{1-\frac{1}{x_{q_{p}^{+}}^{+}}}\right)^{-i g\left(-x_{q^{*}}^{-}+x_{p}^{-}-x_{q^{*}}^{+}+x_{p}^{+}+\left(1 / x_{p}^{-}\right)-\left(1 / x_{q^{*}}^{+}\right)+\left(1 / x_{p}^{+}\right)-\left(1 / x_{q^{*}}^{-}\right)\right)} .
\end{gathered}
$$

In the scaling limit (3.2) we find

$$
\begin{gathered}
a_{2} \simeq \mathcal{O}(1 / \sqrt{\lambda}), \quad a_{6} \simeq 1+\mathcal{O}(1 / \sqrt{\lambda}), \\
a_{1}=\frac{x-X^{-}}{x-X^{+}} \sqrt{\frac{X^{+}}{X^{-}}}+\mathcal{O}(1 / \sqrt{\lambda}),
\end{gathered}
$$

and

$$
\begin{aligned}
\frac{x_{q^{*}}^{-}-x_{p}^{+}}{x_{q^{*}}^{+}-x_{p}^{-}} \frac{1-\frac{1}{1-\frac{1}{x_{q^{*}}^{+} x_{p}^{-}}}}{x_{q^{*}}^{-} x_{p}^{+}} & \sigma^{2}\left(x_{q^{*}}, x_{p}\right) \\
= & \left(\frac{x-X^{+}}{x-X^{-}} \frac{x-1 / X^{+}}{x-1 / X^{-}}\right) e^{-(i 4 \pi(\Delta-J-Q) / \sqrt{\lambda})\left(x /\left(x^{2}-1\right)\right)} \\
& +\mathcal{O}(1 / \sqrt{\lambda}) .
\end{aligned}
$$

For a simple GM we have $X^{ \pm}=1 / X^{\mp}$ and $\sum_{b} S_{b a}^{b a}\left(q^{*}, p\right)(-1)^{F_{b}}$ reduces to the simple result (3.4) in the main text.

\section{APPENDIX C: TWISTS-ORBIFOLDING THE GM}

In this appendix we closely follow the approach of $[16,23,24]$ and most important the one of [25]. The giant magnon solution is a world-sheet soliton whose target space picture is that of a string moving in $S^{2} \subset S^{5}$ with endpoints located on the equator and moving with the speed of light so that [12]

$$
\begin{aligned}
Z(t, x= \pm \infty) & =X_{5}(t, x= \pm \infty)+i X_{6}(t, x= \pm \infty) \\
& =e^{i t \pm i p / 2+i \alpha},
\end{aligned}
$$

\footnotetext{
${ }^{9}$ Note the different sign of the fermion term $-2 a_{6}$ compared to $[10,18]$, which is due to the supertrace. Note, that for the $\mu$-term computation of $[10,18]$ this sign was irrelevant as in their limit the $a_{6}$ term did not contribute. Also, the fact that the supertrace removes the -1 term in $\sum_{b}(-1)^{F_{b}}(S-1)$ (for supersymmetric theories) was neglected there, which however was again irrelevant for the purpose of the evaluation of residues that is necessary for the $\mu$-term. However all these points are crucial for the $F$-term computation in this paper.
} 
where $x$ is the rescaled world-sheet space coordinate which ranges from $-\infty$ to $+\infty$ in the infinite spin limit. Thus, to properly treat the giant magnon we must replace the usual periodic boundary condition by

$$
Z(t, \sigma=2 \pi)=e^{-i p} Z(t, \sigma=0),
$$

with all the other coordinates $X \equiv X_{1}+i X_{2}$ and $Y=X_{3}+$ $i X_{4}$ periodic. The new boundary conditions $(\mathrm{C} 1)$ can be incorporated by a $\mathbb{Z}_{S}$ orbifold with

$$
\mathbf{s}_{3}=\left(-t_{1}+t_{3}, t_{1}-t_{2}+t_{3}, t_{2}\right)=\frac{S}{2 \pi}(0,0,-p),
$$

which for the higher Dynkin diagram Bethe equations used in $[2,23,25]$ amounts to adding a phase,

$$
e^{\left(2 \pi i s_{j} / S\right)}, \quad j=1, \ldots, 7,
$$

[1] M. Staudacher, J. High Energy Phys. 05 (2005) 054.

[2] N. Beisert and M. Staudacher, Nucl. Phys. B727, 1 (2005).

[3] N. Beisert, arXiv:hep-th/0511082.

[4] N. Beisert, R. Hernandez, and E. Lopez, J. High Energy Phys. 11 (2006) 070; N. Beisert, B. Eden, and M. Staudacher, J. Stat. Mech. (2007) P021.

[5] G. Arutyunov, S. Frolov, and M. Zamaklar, J. High Energy Phys. 04 (2007) 002.

[6] J. Ambjorn, R. A. Janik, and C. Kristjansen, Nucl. Phys. B736, 288 (2006).

[7] S. Schafer-Nameki, Phys. Lett. B 639, 571 (2006).

[8] S. Schafer-Nameki and M. Zamaklar, J. High Energy Phys. 10 (2005) 044; S. Schafer-Nameki, M. Zamaklar, and K. Zarembo, J. High Energy Phys. 12 (2006) 020; A. V. Kotikov, L. N. Lipatov, A. Rej, M. Staudacher, and V. N. Velizhanin, J. Stat. Mech. (2007) P10003; G. Arutyunov and S. Frolov, J. High Energy Phys. 12 (2007) 024.

[9] G. Arutyunov, S. Frolov, and M. Zamaklar, Nucl. Phys. B778, 1 (2007).

[10] R. A. Janik and T. Lukowski, Phys. Rev. D 76, 126008 (2007).

[11] M. Luscher, Commun. Math. Phys. 104, 177 (1986); 105, 153 (1986); T. R. Klassen and E. Melzer, Nucl. Phys. B362, 329 (1991).

[12] D. M. Hofman and J. M. Maldacena, J. Phys. A 39, 13095 (2006).

[13] N. Gromov, V. Kazakov, K. Sakai, and P. Vieira, Nucl. Phys. B764, 15 (2007); N. Gromov and V. Kazakov, Nucl. Phys. B780, 143 (2007).

[14] G. Papathanasiou and M. Spradlin, J. High Energy Phys. 06 (2007) 032.

[15] H.-Y. Chen, N. Dorey, and R. F. Lima Matos, J. High Energy Phys. 09 (2007) 106. to the right-hand side of the Bethe equations (in [23] these twists were written as $e^{i \phi_{a}-i \phi_{b}}$, see Eq. (6.1) there). The seven twists $s_{j}$ are given by

$$
\begin{aligned}
\mathbf{s}_{\text {Higher }} & =\left(t_{1}, 0, t_{1}-t_{2}, 2 t_{2}-t_{1}-t_{3}, t_{3}-t_{2}, 0, t_{3}\right) \\
& =\frac{S}{2 \pi}(-p / 2,0,+p / 2,-p,+p / 2,0,-p / 2),
\end{aligned}
$$

which in the language of [23] means

$$
\begin{aligned}
\left(\phi_{1}, \ldots, \phi_{8}\right) & =\left(\phi_{\tilde{1}}, \phi_{\hat{1}}, \phi_{\hat{2}}, \phi_{\tilde{2}}, \phi_{\tilde{3}}, \phi_{\hat{3}}, \phi_{\hat{4}} \phi_{\tilde{4}}\right) \\
& =\left(-\frac{p}{2}, 0,0,-\frac{p}{2},+\frac{p}{2}, 0,0,+\frac{p}{2}\right)
\end{aligned}
$$

thus explaining the choice (2.6) in the main text.
[16] D. Astolfi, V. Forini, G. Grignani, and G. W. Semenoff, Phys. Lett. B 651, 329 (2007).

[17] N. Dorey, J. Phys. A 39, 13119 (2006).

[18] Y. Hatsuda and R. Suzuki, arXiv:0801.0747.

[19] J. A. Minahan and O. O. Sax, arXiv:0801.2064.

[20] V.A. Kazakov, A. Marshakov, J.A. Minahan, and K. Zarembo, J. High Energy Phys. 05 (2004) 024; V. A. Kazakov and K. Zarembo, J. High Energy Phys. 10 (2004) 060; N. Beisert, V. A. Kazakov, and K. Sakai, Commun. Math. Phys. 263, 611 (2006); S. SchaferNameki, Nucl. Phys. B714, 3 (2005); N. Beisert, V. A. Kazakov, K. Sakai, and K. Zarembo, Commun. Math. Phys. 263, 659 (2006); J. High Energy Phys. 07 (2005) 030.

[21] B. Vicedo, J. High Energy Phys. 12, 078 (2007).

[22] J. A. Minahan, A. Tirziu, and A. A. Tseytlin, J. High Energy Phys. 08 (2006) 049.

[23] N. Gromov and P. Vieira, J. High Energy Phys. 04 (2008) 046.

[24] K. Ideguchi, J. High Energy Phys. 09 (2004) 008; A. Solovyov, arXiv:0711.1697.

[25] N. Beisert and R. Roiban, J. High Energy Phys. 11 (2005) 037.

[26] N. Gromov and P. Vieira, Nucl. Phys. B789, 175 (2008).

[27] N. Gromov and P. Vieira, Nucl. Phys. B790, 72 (2008).

[28] R. Roiban, A. Tirziu, and A. A. Tseytlin, J. High Energy Phys. 07 (2007) 056; R. Roiban and A. A. Tseytlin, J. High Energy Phys. 11 (2007) 016; arXiv:0712.2479.

[29] N. Gromov, S. Schafer-Nameki, and P. Vieira (in progress).

[30] G. Arutyunov, S. Frolov, and M. Staudacher, J. High Energy Phys. 10 (2004) 016.

[31] Y. Koma and M. Koma, Nucl. Phys. B713, 575 (2005); Nucl. Phys. B, Proc. Suppl. 140, 329 (2005); arXiv:heplat/0504009. 